\title{
Self-Assembly of Cholesterol-Containing Water-Soluble Polymers
}

\author{
Shin-ichi Yusa \\ Department of Materials Science and Chemistry, University of Hyogo, 2167 Shosha, Himeji, Hyogo 671-2280, Japan \\ Correspondence should be addressed to Shin-ichi Yusa, yusa@eng.u-hyogo.ac.jp
}

Received 15 August 2011; Accepted 29 September 2011

Academic Editor: Eri Yoshida

Copyright (C) 2012 Shin-ichi Yusa. This is an open access article distributed under the Creative Commons Attribution License, which permits unrestricted use, distribution, and reproduction in any medium, provided the original work is properly cited.

Self-assembly of amphiphilic polymers containing cholesteryl groups has proved to be attractive in the field of nanotechnology research. Some cholesterol derivatives are known to form ordered structures which indicate thermotropic and lyotropic liquidcrystalline, monolayers, multilayers, micelles, and liposomes. This paper involves the synthesis and characterization of various kinds of amphiphilic polymers bearing cholesteryl moieties.

\section{Introduction}

Self-assembling water-soluble polymers are of current scientific and technological interest because of their relekvance to biological macromolecular systems and also to various industrial applications [1-3]. Macromolecular self-assemblies can be driven by noncovalent interactions including Coulombic, hydrogen bonding, van der Waals, exchange repulsive, and hydrophobic interactions. Among others, hydrophobic interaction is a major driving force for the selforganization of amphiphilic polymers in water.

Various types of self-assembling amphiphilic polymers have so far been synthesized by various methods. A practical approach to the synthesis of such polymers is to covalently introduce hydrophobes into water-soluble polymers. A large number of hydrophobes can be incorporated into a watersoluble polymer chain by copolymerization of hydrophilic and hydrophobic monomers with a block, alternating, or random sequence distribution. The incorporation of hydrophobic groups into a hydrophilic polymer can alter its solution properties in an aqueous solution. Water forms an organized, ice-like structure around hydrophobic molecules, which is entropically unfavorable $[4,5]$. Therefore, water forces hydrophobic molecules together so that the amount of water structuring is minimized. If the hydrophobic groups are covalently attached to a hydrophilic polymer, associations of hydrophobes either within or between polymer chains occur in water.

The micelle formation of amphiphilic block copolymers in aqueous solutions has been the focus of great interest in a number of excellent reviews [6-8]. It is well established that block copolymers dissolved in a selective solvent-a solvent good for one block and poor for the other-undergo self-organization, leading to the formation of various morphologies, for example, spheres, rods, or lamellae. Water is a selective solvent for amphiphilic block copolymers, allowing for the formation of spherical micelles. Typically, they are formed with hydrophobic cores and hydrophilic outer layers (i.e., shells).

On the other hand, a great deal of effort has also been devoted to the investigations of random copolymers because of their relative ease of synthesis and wide range of monomer selections, compared to block copolymers. A wide variety of self-association phenomena may be anticipated for amphiphilic random copolymers. The self-association is dependent on the type of hydrophobic groups, their content in the copolymer, their sequence distribution, and the type of hydrophilic monomer units.

An important starting point for understanding the selfassembling behavior of hydrophobically modified watersoluble polymers is to know whether polymer-bound hydrophobes associate within the same polymer chain or among different polymer chains in water. The association of polymer-bound hydrophobes in water can occur either within a single polymer chain or between different polymer chains; an intrapolymer association will result in a chain loop while interpolymer association will cross-link (Figure 1).

Figure 2 illustrates an extreme case where all polymerbound hydrophobes undergo interpolymer association. 


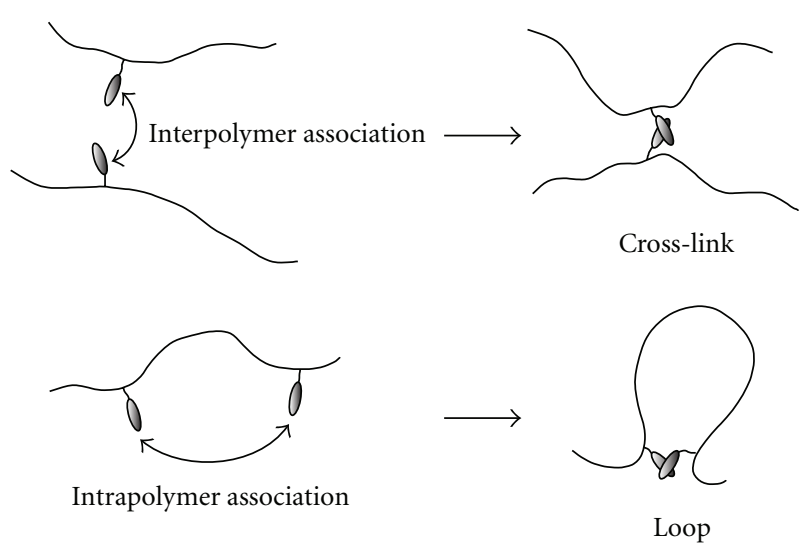

FIGURE 1: Inter- versus intrapolymer hydrophobe association.

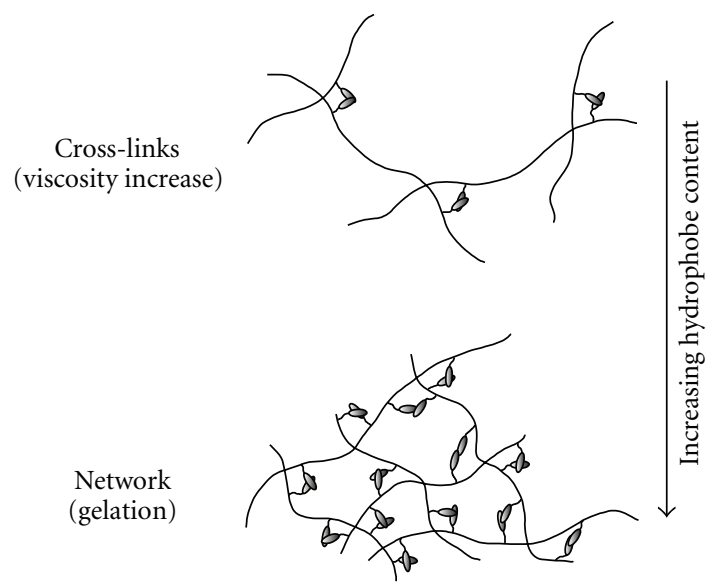

FIGURE 2: Interpolymer hydrophobe association.

When hydrophobe content is low, the interpolymer hydrophobic association may lead to a situation where several polymer chains are cross-linked, causing a large increase in solution viscosity. As the hydrophobe content is increased, infinite networks may be formed, leading to gelation or bulk phase separation.

Figure 3 depicts another extreme case where all polymerbound hydrophobes undergo complete intrapolymer associations. In this extreme case, hydrophobic associations lead to the formation of single molecular self-assemblies or unimolecular micelles. When the content of hydrophobes in a polymer is sufficiently low, a "flower-like" unimolecular micelle may be formed, which consists of a hydrophobic core surrounded by hydrophilic loops shaped into "petals." This type of micelle has been theoretically predicted by Halperin [9] and Semenov et al. [10] for the self-organization of sequential multiblock copolymers in selective solvents. The flower-like micelles can be viewed as a second-order structure formed by a linear polymer chain. As the content of hydrophobes in a copolymer is increased, the size of the hydrophobic core increases and, in turn, the size of the hydrophilic petals decreases. Accordingly, the second-order structure may become unstable because part of the surface of the hydrophobic core is exposed to water. This leads to a further collapse of the second-order structure into a thirdorder structure due to secondary association of hydrophobic cores. Such third-order structures are composed of a number of flower-like micelles collapsed into a highly compact assembly.

Figure 4 shows a case where intrapolymer associations mainly occur, but a portion of hydrophobes undergo interpolymer association. In this situation, intermolecularly bridged flower-like micelles are likely to be formed. The extent of such micellar bridges may depend strongly on the content of hydrophobes in the polymer as well as the polymer concentration. As the content of hydrophobes in a polymer are increased, both the size of the micellar core and the number of micellar bridges would increase. A collapsed micelle network would eventually be formed, giving rise to gelation or bulk phase separation.

Morishima et al. [11-14] have extensively studied hydrophobic associations of amphiphilic random copolymers of sodium 2-(acrylamido)-2-methylpropanesulfonate (AMPS) or sodium acrylate and various hydrophobic comonomers. They have employed, as hydrophobic comonomers, methacrylamides, or methacrylates substituted with various types of hydrophobic groups via various spacer bonds. They have particularly focused on the macromolecular 


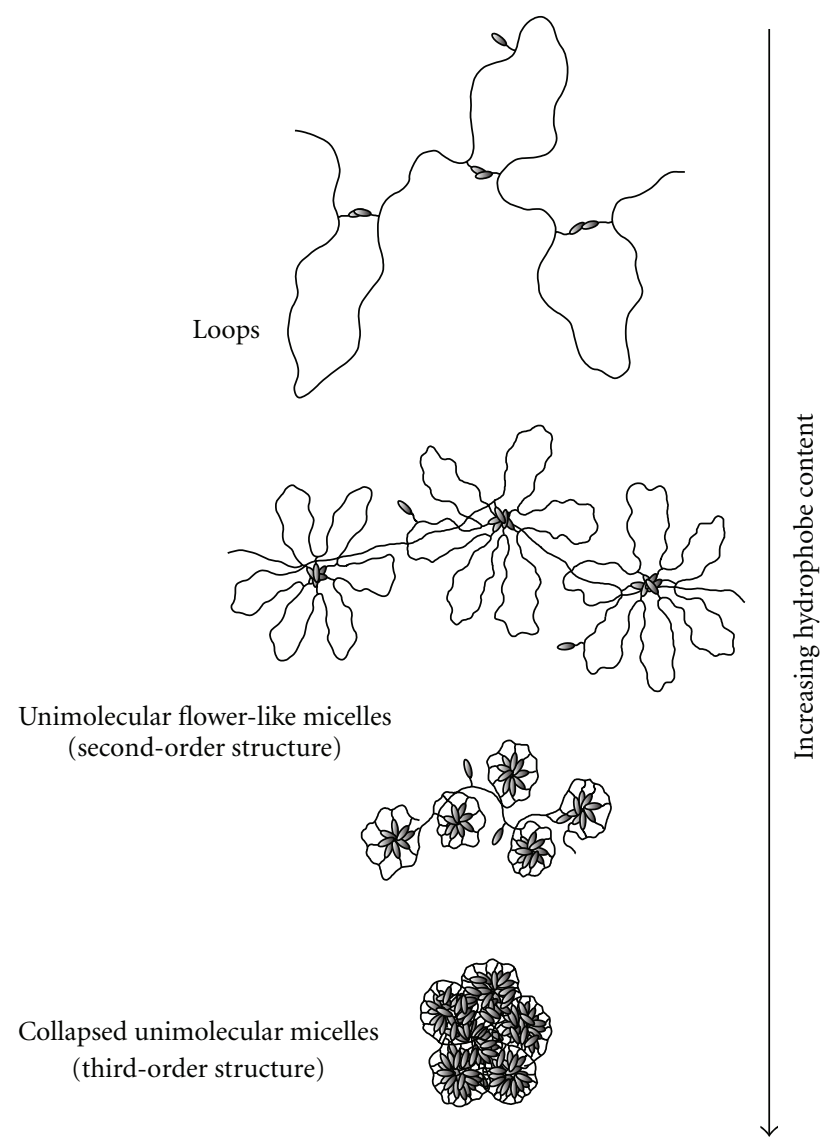

Figure 3: Intrapolymer hydrophobe association.

architecture which results in the two extreme cases, (i) completely intramolecular associations leading to singlemacromolecular self-assemblies independent of polymer concentration and (ii) highly preferential interpolymer associations leading to multipolymeric "transient" networks. From the studies by Morishima et al. $[15,16]$ and others [17-19], it has been revealed that the sequence distribution of charged and hydrophobic units along the polymer chain is a very important structural factor to determine whether the hydrophobic self-association is an intra- or interpolymer event. Block sequences have a strong tendency for interpolymer association, whereas random and alternating sequences tend to associate in an intrapolymer mode. Chang and McCormick [20] have shown that even a subtle difference in the sequence distribution in random copolymers has a considerable effect on the association mode; if the sequence distribution is "block-like" in nature, there is a tendency for intermolecular association.

There is a class of amphiphilic polymers that have hydrophobic moieties at polymer chain ends. If the hydrophobes at chain ends interact strongly, micellar structures may be formed. Figure 5(a) shows schematically the formation of a core-corona type unicore micelle by watersoluble polymers possessing a hydrophobe at one chain end of each polymer. Such micelles may be formed spontaneously at equilibrium between micelles and single free polymer molecules (i.e., unimers) as described by a closed association model [21-23]. A characteristic feature of such equilibrium micelles is that they are nearly monodispersed in mass and size. At a thermodynamic equilibrium, aggregation numbers (numbers of polymer molecules that consist of a single micelle) are determined by the minimum free energy of the system, and therefore the distribution of aggregation numbers around the most stable state is quite narrow [24]. However, if polymers having hydrophobic moieties at both chain ends (doubly end-capped polymers) are present together with the singly end-capped polymers, bridged unicore micelles may be formed (Figure 5(b)). At a very low polymer concentration, a doubly end-capped polymer chain may exist as a looped or open chain-end conformation depending on whether or not the terminal hydrophobes associate in the same polymer chain. These two conformations may exist in equilibrium in aqueous solution and undergo multipolymer association as their own identities. This situation would give rise to the formation of looped coronas and intermicellar bridges. In the case where all polymers are doubly end-capped, polymer chains would form either looped coronas or intermicellar bridges, leading to infinite networks, as conceptually illustrated in Figure 5(c).

Winnik et al. [25, 26] synthesized amphiphilic derivatives of poly( $N$-isopropylacrylamide) (PNIPAM) consisting of a 


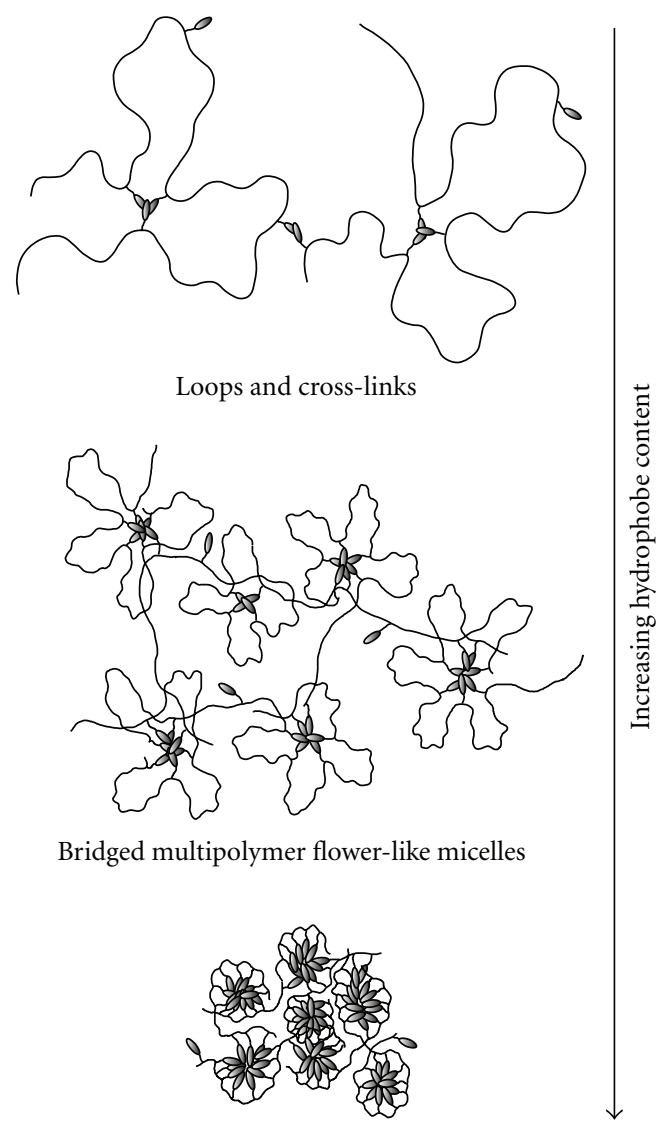

Collapsed bridgedmicelles

FIGURE 4: Inter- plus intrapolymer association.
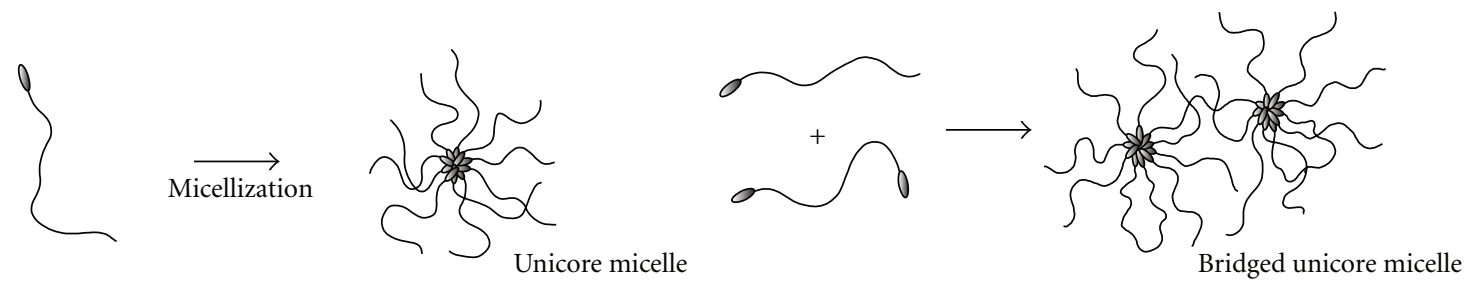

(a)

(b)

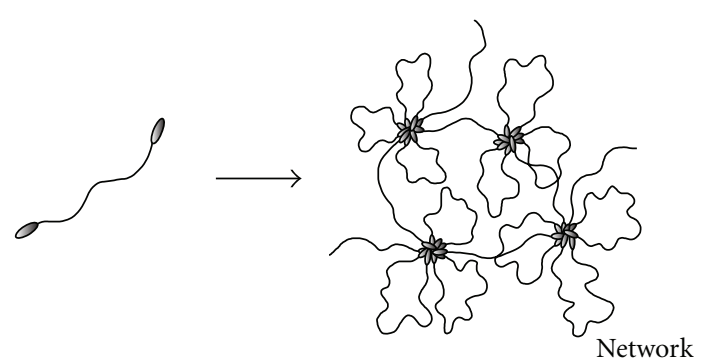

(c)

FIGURE 5: Association of hydrophobically end-modified polymers: (a) singly end-modified polymers; (b) a mixture of singly and doubly end-modified polymers; (c) doubly end-modified polymers. 
PNIPAM chain substituted at one end with a dioctadecyl group by free radical polymerization of $N$-isopropylacrylamide using 4,4'-azobis(4-cyano- $N, N$-dioctadecyl) pentanamide as an initiator. In aqueous solution, the enddioctadecylated PNIPAM forms a multimolecular corecorona type micelle under temperatures below the lower critical solution temperature (LCST). The micelle consists of a core of dioctadecyl chains and corona of solvated PNIPAM chains. Alami et al. [27] reported the aggregation behavior of hydrophobically doubly end-capped poly(ethylene oxide) in aqueous solutions. At very low concentrations, the polymers exist in the form of unimer, some of which are closed to loops, or in small oligomeric aggregates. In semidilute or concentrated regimes, micelle-like polymer aggregates are formed. At higher concentrations, the polymer chains form an infinite network.

\section{Effects of Structural Variations of Amphiphilic Polymers on Association Behavior}

Morishima et al. [28] have shown that (i) the type of hydrophobes, (ii) their content in a polymer, (iii) the sequence distribution of electrolyte and hydrophobic monomer units in a polymer chain, and (iv) the type of spacer bonding between hydrophobes and the polymer chain are particularly important structural parameters determining a preference for intra- or interpolymer hydrophobe association. Random copolymers of AMPS and methacrylamides carrying bulky hydrophobic groups with cyclic structures, such as cyclododecyl, 1-adamantyl, and 1-naphthyl groups, form unimolecular micelles (unimer micelles) in aqueous solutions of a wide range of concentrations if the hydrophobe contents are higher than a certain critical level ( $\geq \mathrm{ca} .20 \mathrm{~mol}$ $\%)$. This phenomenon is due to predominant intramolecular self-association of the hydrophobes with cyclic structure [29-31]. These unimer micelles are very different from the classical surfactant micelles in that (i) all charge and hydrophobes are covalently linked to the polymer backbone, (ii) the unimer micelles are "static" in nature as opposed to the "dynamic" nature of the surfactant micelles which exist in equilibrium between association and dissociation, (iii) the micellar structure is retained even at very low concentrations, and (iv) the unimer micelles remain as such even at very high concentrations.

Random copolymers of AMPS and methacrylamides $N$-substituted with a hydrophobic group such as dodecyl, cyclododecyl, or 1-adamantyl are soluble in water up to about $60 \mathrm{~mol} \%$ of the hydrophobe content. In contrast, random copolymers of AMPS and dodecylmethacrylate are soluble in water only when the content of dodecylmethacrylate is $\leq 15 \mathrm{~mol} \%$. These results indicate that there is a great difference in solubility in water between the polymers with amide and ester spacer bonds connecting hydrophobes to the main chain. Both the amide-spacer and ester-spacer polymers show a tendency for interpolymer association when the hydrophobe contents are lower than about $10 \mathrm{~mol} \%$.
This tendency is much more pronounced in the esterspacer polymers than in the amide-spacer polymers. As the hydrophobe contents in the polymers are increased up to about $20 \mathrm{~mol} \%$ or higher, the amide-spacer polymers show a strong preference for intrapolymer self-association even in a concentrated regime. On the other hand, the esterspacer polymers give strongly turbid solutions when the hydrophobe content is increased to about $15 \mathrm{~mol} \%$.

Since the polymer chain exerts steric constraints to polymer-bound hydrophobes, the degree of the motional and geometrical freedom of polymer-bound hydrophobes has an important effect on their self-association. Therefore, the spacing between hydrophobes and the polymer backbone is a key element to control the hydrophobic association.

\section{Cholesterol-Bearing Pullulan}

Cholesterol (Chol) plays an important role in controlling membrane fluidity in biological systems, arising from its strong tendency for hydrophobic interactions and rigidity of the steroid ring [32]. The hydrophobicity of Chol is stronger than that of other hydrophobic groups such as dodecyl, cyclododecyl, 1-adamantyl, and 1-naphthyl groups. Some Chol derivatives are known to form ordered structures which indicate thermotropic and lyotropic liquid-crystalline (LC), monolayers, multilayers, micelles, and liposomes [33]. Especially, it is well known that comb-like polymers with Chol groups attached to the main chain via a flexible spacer show a thermotropic LC phase [34-36].

Akiyoshi et al. [37-41] have reported that Chol-bearing polysaccharides (Figure 6), especially Chol-bearing pullulan (CHP), formed colloidally stable particles in dilute aqueous solutions when the aqueous solutions were sonicated. A pullulan (weight-average molecular weight $\left(M_{w}\right)=5.5 \times 10^{4}$, molecular weight distribution $\left.\left(M_{w} / M_{n}\right)=1.6\right)$ derivative carrying two Chol groups per 100 glucose units was prepared, which was characterized by size exclusion column chromatography (SEC), dynamic light scattering (DLS), static light scattering (SLS), transmission electron microscopy (TEM), ${ }^{1} \mathrm{H} \mathrm{NMR}$, and fluorescence spectroscopy. SEC results indicated that $\mathrm{CHP}$ intermolecularly aggregates and provides relatively monodispersive particles upon ultrasonication. Spherical particles with relatively uniform size (the diameter $=25 \pm 5 \mathrm{~nm}$ ) can be observed in the negatively stained TEM of CHP. The hydrodynamic radius $\left(R_{h}\right)$ of the CHP self-aggregate estimated from DLS is approximately $13 \mathrm{~nm}$, and the aggregation number determined by SLS was approximately $13 ; M_{w}$ of the self-aggregate was $7.6 \times 10^{6}$, the radius of gyration $\left(R_{g}\right)$ was $16.8 \mathrm{~nm}$, and the second virial coefficient $\left(A_{2}\right)$ was $2.6 \times 10^{-4} \mathrm{~mol} / \cdot \mathrm{mL} / \mathrm{g}^{2}$. The mean aggregation number of the Chol moieties $\left(N_{\mathrm{Chol}}\right)$ can be estimated by fluorescence quenching experiments to be $4.2 \pm 0.5$. The CHP self-aggregate carries about 59 Chol moieties, which corresponds to 14 times the mean aggregation number of the Chol moieties. This suggests that approximately 14 independent domains are distributed in one nanoparticle. The critical aggregation concentration of CHP was $0.01 \mathrm{~g} / \mathrm{L}$, estimated from fluorescence 


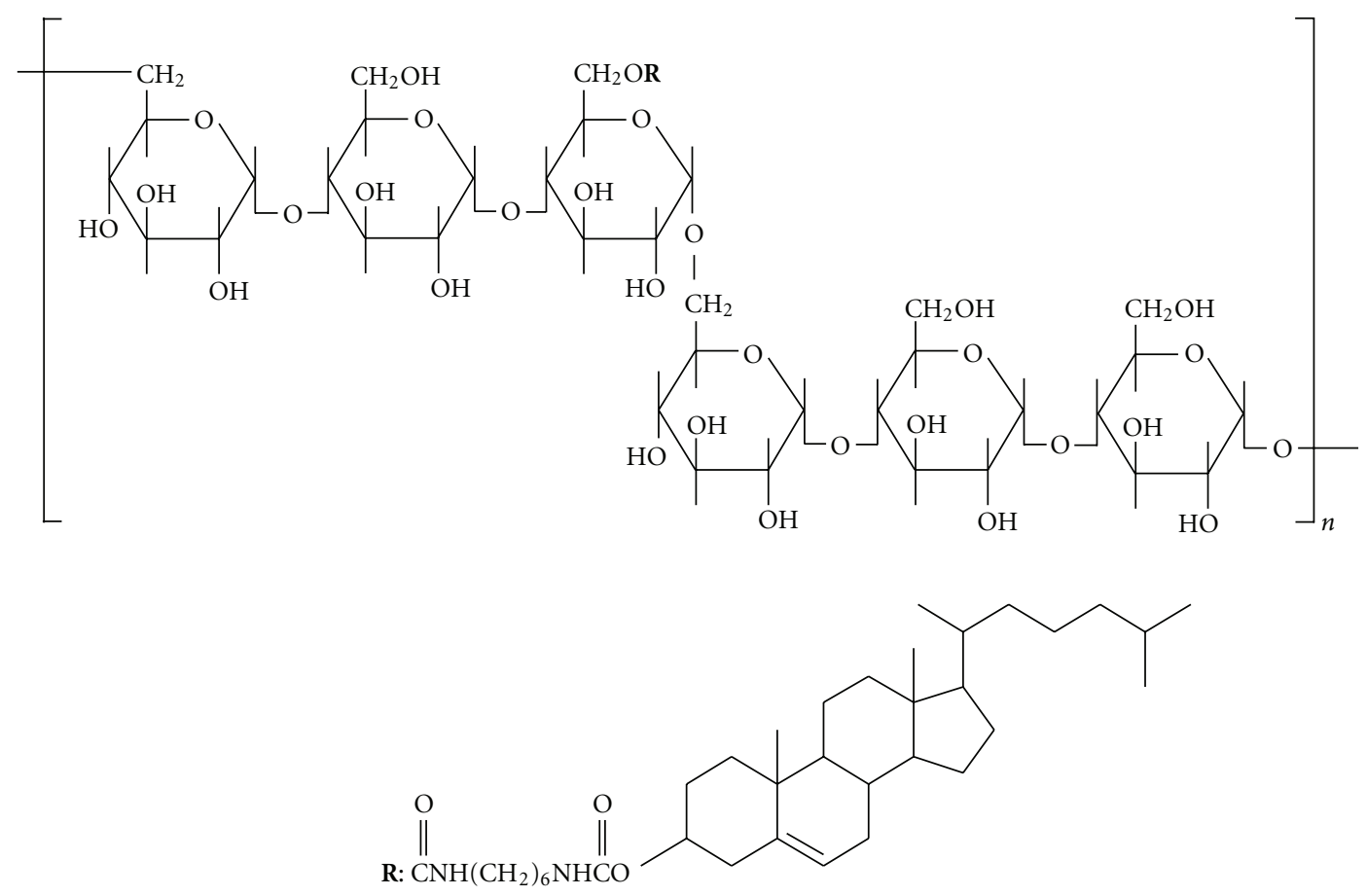

FIGURE 6: Chemical structure of cholesterol-bearing pullulan (CHP).

measurements using $N$-phenyl-1-naphtylamine (PNA) as a fluorescence probe. CHP shows no surface activity at all up to the concentration of $0.145 \mathrm{~g} / \mathrm{L}$. Existence of microdomains consisting of both the rigid core of hydrophobic Chol and the relatively hydrophilic polysaccharide shell was suggested by the line broadening of ${ }^{1} \mathrm{H}$ NMR proton signals of Chol moieties in CHP, and the incorporation of several hydrophobic fluorescence probes in the CHP aggregates. The CHP aggregate by themselves in water and provide colloidally stable and monodispersed nanoparticles above the critical concentration. The CHP self-aggregate is crucial for making a stable complex with various hydrophobic and hydrophilic substances. The main driving force of the complexation is the hydrophobic interactions. The particle size varies with the substitution degree of Chol. However, the association number of polymer chains is about ten, independent of the Chol content.

These studies suggest that, because of a strong tendency for self-association of $\mathrm{Chol}$ as well as its structural rigidity, a polyelectrolyte may be modified into a strongly associative polymer by covalently incorporating a small amount of Chol moieties into the polymer chain.

\section{Polyelectrolyte-Containing Pendant Cholesteryl Moieties}

Chol moieties-containing polyanion ( $\mathrm{P}(\mathrm{A} / \mathrm{ChM}-5)$, Figure 7) was synthesized via ordinary random radical copolymerization of sodium 2-(acrylamido)-2-methylpropanesulfonate (AMPS) and cholesteryl 6-methacryloyloxyhecanoate (ChM-5) [42, 43]. Copolymerization of varying molar ratios of AMPS and ChM-5 were performed in the presence of radical initiator in $N, N$-dimethylformamide (DMF) at $60^{\circ} \mathrm{C}$, and the copolymers with ChM-5 contents ranging from 0.5 to $10 \mathrm{~mol} \%$ were prepared. The copolymers with ChM-5 contents $<5 \mathrm{~mol} \%$ can be completely soluble in water. ${ }^{1} \mathrm{H}$ NMR spectra for $\mathrm{P}(\mathrm{A} / \mathrm{ChM}-5)$ were measured in deuterium oxide and deuterated dimethyl sulfoxide (DMSO$d_{6}$ ) at room temperature. In deuterium oxide, resonance peaks due to Chol protons are not observed because of considerable line broadening. In DMSO- $d_{6}$, by contrast, no such line broadening was observed in the Chol resonance peaks. These results indicate that motions of Chol moieties in $\mathrm{P}(\mathrm{A} / \mathrm{ChM}-5)$ are highly restricted in deuterium oxide, arising from hydrophobic self-association of Chol groups. The $M_{w}$ values $\mathrm{P}(\mathrm{A} / \mathrm{ChM}-5)$ with various $\mathrm{ChM}-5$ contents are estimated by SEC. The interpolymer Chol association is absent in the water and acetonitrile (50/50) mixed solvent, and the molar masses of all the random copolymers are more or less the same. In contrast, the $M_{w}$ values for $\mathrm{P}(\mathrm{A} / \mathrm{ChM}-5)$ estimated by SLS in $0.1 \mathrm{M} \mathrm{NaCl}$ aqueous solution are much greater than those estimated by SEC using the water and acetonitrile mixed solvent. The apparent $M_{w}$ values for the random copolymers with 1 and $5 \mathrm{~mol} \%$ ChM- 5 estimated by SLS are $4.93 \times 10^{5}$ and $1.90 \times 10^{6}$, respectively. The $R_{g}$ values for the random copolymer with 1 and $5 \mathrm{~mol} \%$ ChM-5 are 50.3 and $65.7 \mathrm{~nm}$, respectively. The $R_{h}$ distributions measured by DLS for the random copolymers with 1 and 5 mol \% ChM-5 shows bimodal and unimodal distributions, respectively. The small and large $R_{h}$ values for $\mathrm{P}(\mathrm{A} / \mathrm{ChM}-5)$ with $1 \mathrm{~mol} \% \mathrm{ChM}-5$ are attributed to nonassociated polymers, that is, unimers and interpolymer 


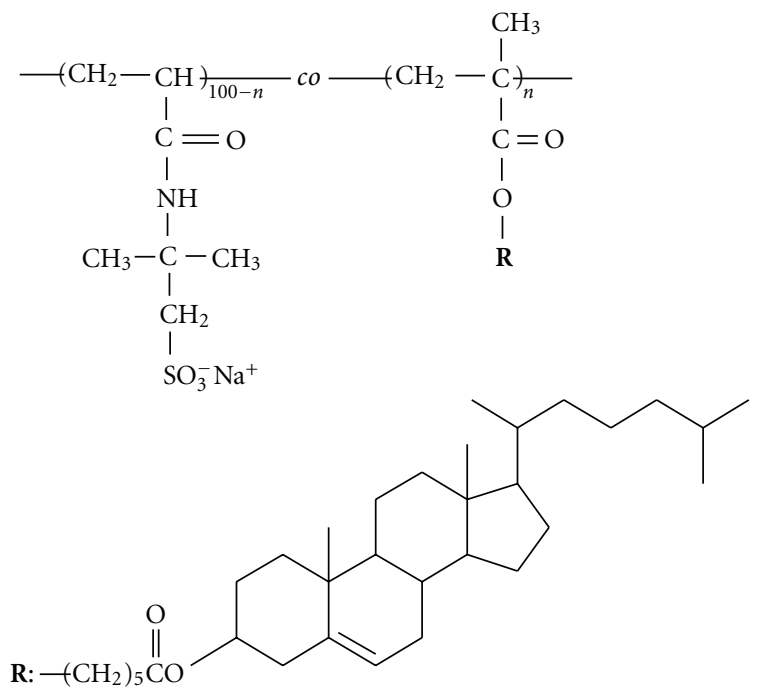

FIGURE 7: Chemical structure of cholesterol-bearing polyelectrolyte.

associations in water. $\mathrm{P}(\mathrm{A} / \mathrm{ChM}-5)$ with $5 \mathrm{~mol} \% \mathrm{ChM}-5$ can be observed at only large $R_{h}$ values, indicating that most polymer chains are intermolecularly associated in water. The aggregation number for $\mathrm{P}(\mathrm{A} / \mathrm{ChM}-5)$ with $5 \mathrm{~mol} \%$ ChM-5 can be roughly calculated to be approximately 50 from the molecular weight determined by SLS and SEC.

All the results just presented led us to propose a conceptual model for interpolymer aggregated formed by $\mathrm{P}(\mathrm{A} / \mathrm{ChM}-5)$ with $5 \mathrm{~mol} \% \mathrm{ChM}-5$ in water. The Chol pendants in the random copolymer associate both intra- and intermolecularly. Intrapolymer association leads to a flowerlike micelle $[9,10]$ in which a hydrophobic microdomainformed from Chol association-is surrounded by loops of polyanion segments. The concurrent interpolymer Chol association will link the flower-like micelle together, and thus intermolecularly-bridged flower-like micelles are formed. Generally, small amounts of large-size hydrophobic groups introduced into side chains in water-soluble polymers apt to associate intermolecularly.

The average number of Chol groups contained in one microdomain, that is, the average aggregation number of Chol groups $\left(N_{\text {chol }}\right)$, is determined by a time resolved fluorescence quenching technique. For this experiment, $\mathrm{P}(\mathrm{A} / \mathrm{ChM}$ 5) with $5 \mathrm{~mol} \% \mathrm{ChM}-5$, pyrene as a fluorescence probe, and 3,4-dimethylbenzophenoe (DMBP) as a fluorescence quencher were used. The number of polymer chains composed of one intermolecularly bridged flower-like micelle, that is, the apparent aggregation number $\left(N_{\mathrm{agg}}\right)$ of the polymer chains of $\mathrm{P}(\mathrm{A} / \mathrm{ChM}-5)$ with $5 \mathrm{~mol} \% \mathrm{ChM}-5$, was roughly estimated based on molecular weight to be about 50 . $\mathrm{P}(\mathrm{A} / \mathrm{ChM}-5)$ with $5 \mathrm{~mol} \% \mathrm{ChM}-5$ possesses on average 3.5 Chol groups, as calculated from the molecular weight and the Chol content. Therefore, the number of Chol groups in the multipolymer associate is roughly estimated to be 175 , consisting of 10 cross-linking sites with each site consisting 17-19 Chol moieties.

\section{Cholesterol-End-Capped Polyelectrolyte}

Chol-substituted azo initiator, 4,4-azobis(4-cyano-1-cholesteryl)pentanoate (AzCCP) was prepared [44]. Free radical polymerization of AMPS using AzCPP as an initiator was performed to obtain a Chol-end-capped polymer (CholPAMPS, Figure 8). The number-average degree of polymerization (DP) of Chol-PAMPS is estimated to be about 72 based on a ${ }^{1} \mathrm{H}$ NMR spectrum measured in DMSO$d_{6}$ by assuming all the chain termination to occur via disproportionation. Using the DP estimated from ${ }^{1} \mathrm{H}$ NMR as a basis and assuming each polymer chain has one Chol group at the polymer chain end, the DP estimated from SEC was found to be very similar. It is anticipated that CholPAMPS would form a spherical core-shell type micelle with a Chol aggregate in the core and PAMPS chains in the shell. If that is the case, $R_{h}$ of the micelle should be equal to or smaller than the length of the fully extended Chol-PAMPS chain. For Chol-PAMPS of DP $=72$, this length is approximately $20 \mathrm{~nm}$. However, $R_{h}$ values observed by DLS in the various polymer concentrations are of the order of $50 \mathrm{~nm}$, and this size is obviously too large for the core-shell micelle. It is concluded that the multipolymer aggregates of Chol-PAMPS are not spherical core-shell micelles. There is a possibility that some polymer chains possess Chol groups at both chain ends, which are produced by the recombination of growing chain radicals or by primary radical termination. Even if most of the polymers have one Chol group at one chain end and they form spherical micelles, a small number of polymer possessing Chol groups at both ends may bridge the micelles, leading to an increase in hydrodynamic size. Furthermore, it seems reasonable to consider that this bridging is more likely to occur at high polymer concentrations, thus leading to a large increase in $R_{h}$. Fluorescence emission and excitation spectra for pyrene probe solubilized in the aggregates of Chol-PAMPS suggested the presence of the critical micelle concentration $(\mathrm{cmc})$ around $0.6 \mathrm{~g} / \mathrm{L}$ in water. 


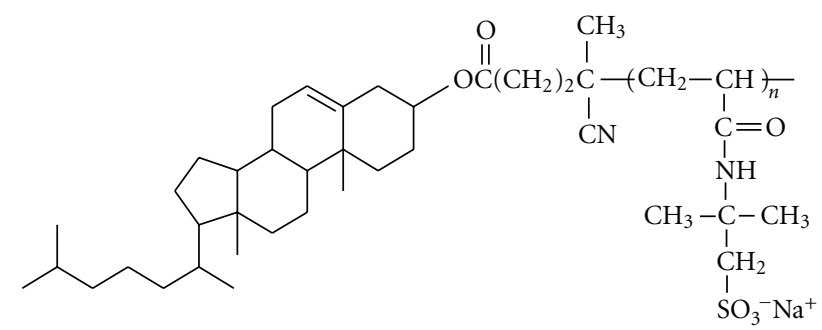

FIGURE 8: Chemical structure of cholesterol-end-capped polyelectrolyte.

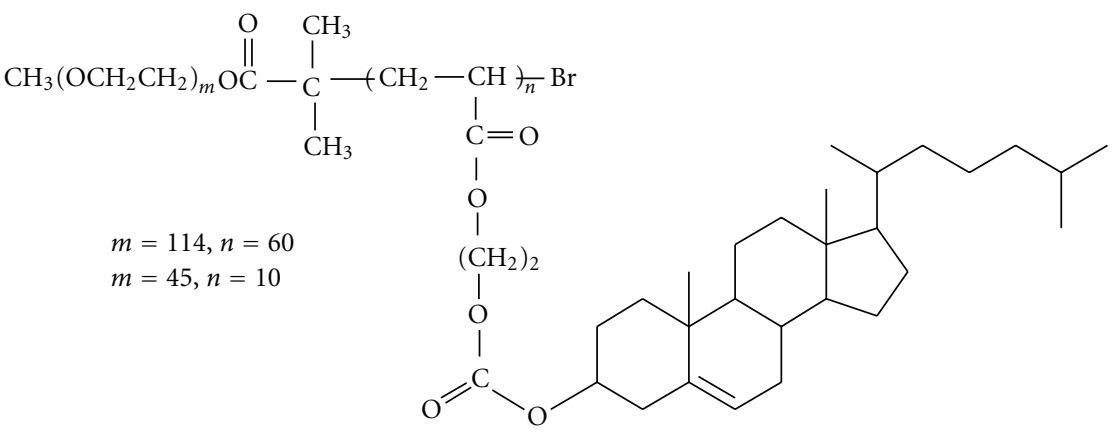

FIGURE 9: Chemical structure of amphiphilic block copolymers containing a Chol-based block.

\section{Amphiphilic Block Copolymer Containing Cholesterol-Bearing Block}

An amphiphilic block copolymer is a typical synthetic system that reveals a controlled balance of amphiphilicity and can assume various well-organized architectures, including spherical, rod, and lamellar structures $[45,46]$. In the past decade, the studies on self-assembly of block copolymers in solution have been extensively pursued [47, 48]. Figure 9 shows the chemical structure of the amphiphilic LC diblock copolymers, PEG5000-b-PAChol(14/86) and PEG2000- $b$ PAChol(28/72), which are prepared by a typical atom transfer radical polymerization (14/86 and 28/72 are the hydrophilic/hydrophobic weight ratios) [49]. These diblock copolymers present LC mesophase measured by differential scanning calorimetry (DSC). X-ray scattering experiments on a bulk sample of PEG5000- $b$-PAChol(14/86) revealed a smectic A phase with lamellar period $(d)$ of $4.29 \mathrm{~nm}$. This period corresponds to a value being the wholly extended length of the Chol moieties. For PEG2000- $b$-PAChol(28/72) in the same position of reflection $(d=4.3 \mathrm{~nm})$, only diffuse layer reflection is observed, which corresponds to a fluctuation of smectic structure in the LC block. The mesophase in the LC part is a chiral nematic from the polarizing microscope observation. Self-assembly of the amphiphilic block copolymers in water was performed. Nanofibers with a lamellar fine structure were formed in water by the block copolymer PEG5000- $b$-PAChol(14/86). The lamellar structure in their hydrophobic core has the same origin as that of the smectic A phase observed in the bulk sample. Both of them result from the interdigital smectic A self-organization of the Chol-based mesogen. On the other hand, polymer vesicles instead of nanofibers are formed by PEG2000- $b$-PAChol(28/72), in bulk sample of which only a nematic phase with smectic fluctuation is observed.

\section{Conclusion}

Polymer-bound Chol moieties show a strong tendency for self-association in water. The strongly stacking nature and hydrophobically associating nature of Chol groups incorporated in polymers would allow one to design a variety of associative polymers that form a variety of nanoorganized structures. These Chol-bearing associative polymers may find usefulness in studies of interactions with lipid membranes as well as potentials for practical applications such as chemical delivery systems.

\section{Acronyms}

AMPS: Sodium 2-(acrylamido)-2methylpropanesulfonate

PNIPAM: Poly(N-isopropylacrylamide)

LCST: Lower critical solution temperature

Chol: Cholesterol

LC: $\quad$ Liquid crystalline

CHP: Cholesterol-bearing pullulan

$M_{w}$ : Weight-average molecular weight

$M_{w} / M_{n}$ : Molecular weight distribution

SEC: $\quad$ Size exclusion column chromatography

DLS: Dynamic light scattering

SLS: $\quad$ Static light scattering

TEM: Transmission electron microscopy

${ }^{1} \mathrm{H}$ NMR: Proton nuclear magnetic resonance 


\begin{tabular}{|c|c|}
\hline$R_{h}:$ & Hydrodynamic radius \\
\hline$R_{g}:$ & Radius of gyration \\
\hline$A_{2}:$ & Second virial coefficient \\
\hline$N_{\text {Chol }}:$ & $\begin{array}{l}\text { Aggregation number of cholesteryl } \\
\text { moieties }\end{array}$ \\
\hline PNA: & $N$-Phenyl-1-naphtylamine \\
\hline $\mathrm{P}(\mathrm{A} / \mathrm{ChM}-5)$ : & $\begin{array}{l}\text { Poly(sodium 2-(acrylamido)-2- } \\
\text { methylpropanesulfonate-co- } \\
\text { cholesteryl } \\
\text { 6-methacryloyloxyhecanoate) }\end{array}$ \\
\hline ChM-5: & $\begin{array}{l}\text { Cholesteryl } \\
6 \text {-methacryloyloxyhecanoate }\end{array}$ \\
\hline DMF: & $N, N$-Dimethylformamide \\
\hline DMSO- $d_{6}:$ & Deuterated dimethyl sulfoxide \\
\hline DMBP: & 3,4-Dimethylbenzophenoe \\
\hline$N_{\text {agg }}:$ & Aggregation number \\
\hline AzCCP: & $\begin{array}{l}\text { 4,4-Azobis }(4 \text {-cyano- } 1 \text { - } \\
\text { cholesteryl)pentanoate }\end{array}$ \\
\hline Chol-PAMPS: & $\begin{array}{l}\text { Cholesterol-end-capped poly(sodium } \\
\text { 2-(acrylamido)-2- } \\
\text { methylpropanesulfonate) }\end{array}$ \\
\hline DP: & $\begin{array}{l}\text { Number-average degree of } \\
\text { polymerization }\end{array}$ \\
\hline cmc: & Critical micelle concentration \\
\hline PEG5000-b-PAChol: & $\begin{array}{l}\text { Poly(ethylene } \\
\text { glycol) } 5000 \text {-block-poly(cholesteryl } \\
\text { acryloyloxy ethyl carbonate) }\end{array}$ \\
\hline PEG2000- $b$-PAChol: & $\begin{array}{l}\text { Poly(ethylene } \\
\text { glycol)2000-block-poly(cholesteryl } \\
\text { acryloyloxy ethyl carbonate) }\end{array}$ \\
\hline $\begin{array}{l}\text { DSC: } \\
d:\end{array}$ & $\begin{array}{l}\text { Differential scanning calorimetry } \\
\text { Lamellar period. }\end{array}$ \\
\hline
\end{tabular}

\section{References}

[1] C. L. McCormick, J. Bock, and D. N. Schulz, Encyclopedia of Polymer Science and Engineering, John Wiley and Sons, New York, NY, USA, 1989.

[2] P. Dubin, J. Bock, R. M. Davies, D. N. Schulz, and C. Thies, Macromolecular Complexes in Chemistry and Biology, Springer, Berlin, Germany, 1994.

[3] J. E. Glass, Polymers in Aqueous Media: Performance through Association, Advances in Chemistry Series 223, American Chemical Society, Washington, DC, USA, 1989.

[4] G. Némethy and H. A. Scheeaga, "Structure of water and hydrophobic bonding in proteins. I. A model for the thermodynamic properties of liquid water," The Journal of Chemical Physics, vol. 36, no. 12, pp. 3382-3400, 1962.

[5] G. Némethy and H. A. Scheraga, "Structure of water and hydrophobic bonding in proteins. II. Model for the thermodynamic properties of aqueous solutions of hydrocarbons," The Journal of Chemical Physics, vol. 36, no. 12, pp. 3401-3417, 1962.

[6] A. Halperin, M. Tirrell, and T. P. Lodge, "Tethered chains in polymer microstructures," Advances in Polymer Science, vol. 100, pp. 30-71, 1991.

[7] Y. Morishima, "Unimolecular micelles of hydrophobically modified polyelectrolytes," in Solvents and Self-Organization of Polymers, S. E. Webber, D. Tuzar, and P. Munk, Eds., pp. 331-358, Kluwer Academic Publishers, Dordrecht, The Netherlands, 1996.

[8] S. E. Webber, "Polymer micelles: an example of self-assembling polymers," Journal of Physical Chemistry B, vol. 102, no. 15, pp. 2618-2626, 1998.

[9] A. Halperin, "On the collapse of multiblock copolymers," Macromolecules, vol. 24, no. 6, pp. 1418-1419, 1991.

[10] A. N. Semenov, J. F. Joanny, and A. R. Khokhlov, "Associating polymers: equilibrium and linear viscoelasticity," Macromolecules, vol. 28, no. 4, pp. 1066-1075, 1995.

[11] M. W. Urban and T. Provder, Multidimensional Spectroscopy of Polymers: Vibrational, NMR, and Fluorescence Techniques, ACS Symposium Series 598, American Chemical Society, Washington, DC, USA, 1995.

[12] Y. Morishima, S. Nomura, T. Ikeda, M. Seki, and M. Kamachi, "Characterization of unimolecular micelles of random copolymers of sodium 2-(acrylamido)-2-methylpropanesulfonate and methacrylamides bearing bulky hydrophobic substituents," Macromolecules, vol. 28, no. 8, pp. 2874-2881, 1995.

[13] M. Seki, Y. Morishima, and M. Kamachi, "Characterization of the complexes of amphiphilic polyanions and double-chain cationic surfactants," Macromolecules, vol. 25, no. 24, pp. 6540-6546, 1992.

[14] Y. Morishima, M. Tsuji, M. Seki, and M. Kamachi, "Synthesis, NMR relaxation, and photoisomerization of amphiphilic polyelectrolytes covalently tethered with azobenzene moieties having bulky hydrophobic substituents," Macromolecules, vol. 26, no. 13, pp. 3299-3305, 1993.

[15] K. Kamioka, S. E. Webber, and Y. Morishima, "Solvent dependence of energy trapping in methacrylic acid-vinylphenanthrene block copolymers," Macromolecules, vol. 21, no. 4, pp. 972-978, 1988.

[16] Y. Morishima, H. S. Lim, S. I. Nozakura, and J. L. Sturtevant, "Effect of monomer sequence distribution in 2vinylnaphthalene-maleic acid copolymers on energy migration excimer formation in aqueous solution," Macromolecules, vol. 22, no. 3, pp. 1148-1154, 1989.

[17] C. L. McCormick and Y. Chang, "Water-soluble copolymers. 58. Associative interactions and photophysical behavior of amphiphilic terpolymers prepared by modification of maleic anhydride/ethyl vinyl ether copolymers," Macromolecules, vol. 27, no. 8, pp. 2151-2158, 1994.

[18] K. Prochazka, D. Kiserow, C. Ramireddy, Z. Tuzar, P. Munk, and S. E. Webber, "Time-resolved fluorescence studies of the chain dynamics of naphthalene-labeled polystyrene-blockpoly(methacrylic acid) micelles in aqueous media," Macromolecules, vol. 25, no. 1, pp. 454-460, 1992.

[19] C. L. McCormick and L. C. Salazar, "Water soluble copolymers: 46. Hydrophilic sulphobetaine copolymers of acrylamide and 3-(2-acrylamido-2-methylpropanedimethylammonio)-1-propanesulphonate," Polymer, vol. 33, no. 21, pp. 4617-4624, 1992.

[20] Y. Chang and C. L. McCormick, "Water-soluble copolymers. 49. Effect of the distribution of the hydrophobic cationic monomer dimethyldodecyl(2-acrylamidoethyl)ammonium bromide on the solution behavior of associating acrylamide copolymers," Macromolecules, vol. 26, no. 22, pp. 6121-6126, 1993.

[21] H. G. Elias, "Nonionic micelles," Journal of Macromolecular Science: Part A, vol. 7, no. 3, pp. 601-622, 1973.

[22] Z. Tuzar, P. Kratochvíl, K. Procházka, and P. Munk, "Block copolymer micelles in aqueous media," Collection of Czechoslovak Chemical Communications, vol. 58, no. 10, pp. 2362-2369, 1993. 
[23] E. Matijevic, Surface and Colloid Science Series, vol. 51, Plenum, New York, NY, USA, 1993.

[24] M. Tian, A. Qin, C. Ramireddy et al., "Hybridization of block copolymer micelles," Langmuir, vol. 9, no. 7, pp. 1741-1748, 1993.

[25] F. M. Winnik, A. R. Davidson, G. K. Hamer, and H. Kitano, "Amphiphilic poly(N-isopropylacrylamides) prepared by using a lipophilic radical initiator: synthesis and solution properties in water," Macromolecules, vol. 25, no. 7, pp. 18761880, 1992.

[26] A. Yamazaki, J. M. Song, F. M. Winnik, and J. L. Brash, "Synthesis and solution properties of fluorescently labeled amphiphilic (N-alkylacrylamide) oligomers," Macromolecules, vol. 31, no. 1, pp. 109-115, 1998.

[27] E. Alami, M. Almgren, W. Brown, and J. François, "Aggregation of hydrophobically end-capped poly(ethylene oxide) in aqueous solutions. Fluorescence and light-scattering studies," Macromolecules, vol. 29, no. 6, pp. 2229-2243, 1996.

[28] Y. Morishima, Y. Tominaga, M. Kamachi, T. Okada, Y. Hirata, and N. Mataga, "Photoinduced charge separation by chromophores encapsulated in the hydrophobic compartment of amphiphllic polyelectrolytes with various aliphatic hydrocarbons," Journal of Physical Chemistry, vol. 95, no. 15, pp. 6027-6034, 1991.

[29] Y. Morishima, M. Tsuji, M. Kamachi, and K. Hatada, "Photochromic isomerization of azobenzene moieties compartmentalized in hydrophobic microdomains in a microphase structure of amphiphilic polyelectrolytes," Macromolecules, vol. 25, no. 17, pp. 4406-4410, 1992.

[30] Y. Morishima, Y. Tominaga, S. Nomura, M. Kamachi, and T. Okada, "Rapid migration and trapping of photoexcited electronic energy in amphiphilic polyelectrolytes containing naphthalene chromophores and pyrene traps," Journal of Physical Chemistry, vol. 96, no. 4, pp. 1990-1994, 1992.

[31] H. Aota, Y. Morishima, and M. Kamachi, "Compartmentalization of zinc (II) tetraphenylporphyrin in a hydrophobic microdomain of an amphiphilic polyelectrolyte: a physicochemical model of biological metalloporphyrin systems," Photochemistry and Photobiology, vol. 57, no. S1, pp. 989-995, 1993.

[32] R. A. Demel and B. De Kruyff, "The function of sterols in membranes," Biochimica et Biophysica Acta, vol. 457, no. 2, pp. 109-132, 1976.

[33] H. Ringsdorf, B. Schlarb, and J. Venzmer, "Molecular architecture and function of polymeric oriented systems: models for the study of organization, surface recognition, and dynamics of biomembrnaes," Angewandte Chemie International, vol. 27, no. 1, pp. 113-158, 1988.

[34] V. P. Shibaev, N. A. Platé, and Y. S. Freidzon, "Thermotropic liquid crystalline polymers. I. Cholesterol-containing polymers and copolymers," Journal of Polymer Science, vol. 17, no. 6, pp. 1655-1670, 1979.

[35] V. P. Shibaev, R. V. Tal'roze, F. I. Karakhanova, and N. A. Platé, "Thermotropic liquid crystalline polymers. II. Polymers with amino acid fragments in the side chains," Journal of Polymer Science, vol. 17, no. 6, pp. 1671-1684, 1979.

[36] T. Yamaguchi, T. Asada, H. Hayashi, and N. Nakamura, "Dependence of the packing structure of mesogenic groups on the flexible spacer length of liquid crystalline side-chain polymers," Macromolecules, vol. 22, no. 3, pp. 1141-1144, 1989.

[37] K. Akiyoshi, K. Nagai, T. Nishikawa, and J. Sunamoto, "Selfaggregates of hydrophobized polysaccharide as a host for macromolecular guests," Chemistry Letters, vol. 21, no. 9, pp. 1727-1730, 1992.

[38] K. Akiyoshi, S. Deguchi, N. Moriguchi, S. Yamaguchi, and J. Sunamoto, "Self-aggregates of hydrophobized polysaccharides in water. Formation and characteristics of nanoparticles," Macromolecules, vol. 26, no. 12, pp. 3062-3068, 1993.

[39] S. Deguchi, K. Akiyoshi, and J. Sunamoto, "Solution property of hydrophobized pullulan conjugated with poly(ethylene oxide)-poly(propylene oxide)-poly(ethylene oxide) block copolymer. Formation of nanoparticles and their thermosensitivity," Macromolecular Rapid Communications, vol. 15, no. 9, pp. 705-711, 1994.

[40] T. Nishikawa, K. Akiyoshi, and J. Sunamoto, "Supramolecular assembly between nanoparticles of hydrophobized polysaccharide and soluble protein complexation between the self-aggregate of cholesterol-bearing pullulan and $\alpha$ chymotrypsin," Macromolecules, vol. 27, no. 26, pp. 76547659, 1994.

[41] K. Akiyoshi, S. Deguchi, H. Tajima, T. Nishikawa, and J. Sunamoto, "Self-assembly of hydrophobized polysaccharide. Structure of hydrogel nanoparticle and complexation with organic compounds," Proceedings of the Japan Academy B, vol. 71, no. 1, pp. 15-19, 1995.

[42] S. I. Yusa, M. Kamachi, and Y. Morishima, "Hydrophobic self-association of cholesterol moieties covalently linked to polyelectrolytes: effect of spacer bond," Langmuir, vol. 14, no. 21, pp. 6059-6067, 1998.

[43] S. I. Yusa, A. Hashidzume, and Y. Morishima, "Interpolymer association of cholesterol pendants linked to a polyelectrolyte as studied by quasielastic light scattering and fluorescence techniques," Langmuir, vol. 15, no. 26, pp. 8826-8831, 1999.

[44] S. I. Yusa, M. Kamachi, and Y. Morishima, "Self-association of cholesterol-end-capped poly(sodium 2-(acrylamido)2-methylpropanesulfonate) in aqueous solution," Macromolecules, vol. 33, no. 4, pp. 1224-1231, 2000.

[45] B. Lindman and P. Alexandridis, Amphiphilic Block Copolymer, Elsevier, Amsterdam, The Netherlands, 2000.

[46] I. W. Hamley, Block Copolymers in Solution, John Wiley and Sons, New York, NY, USA, 2005.

[47] D. E. Discher and A. Eisenberg, "Polymer vesicles," Science, vol. 297, no. 5583, pp. 967-973, 2002.

[48] H. Kukula, H. Schlaad, M. Antonietti, and S. Förster, "The formation of polymer vesicles or "peptosomes" by polybutadiene-block-poly(L-glutamate)s in dilute aqueous solution," Journal of the American Chemical Society, vol. 124, no. 8, pp. 1658-1663, 2002.

[49] R. Piñol, L. Jia, F. Gubellini et al., "Self-assembly of PEGb-liquid crystal polymer: the role of smectic order in the formation of nanofibers," Macromolecules, vol. 40, no. 16, pp. 5625-5627, 2007. 

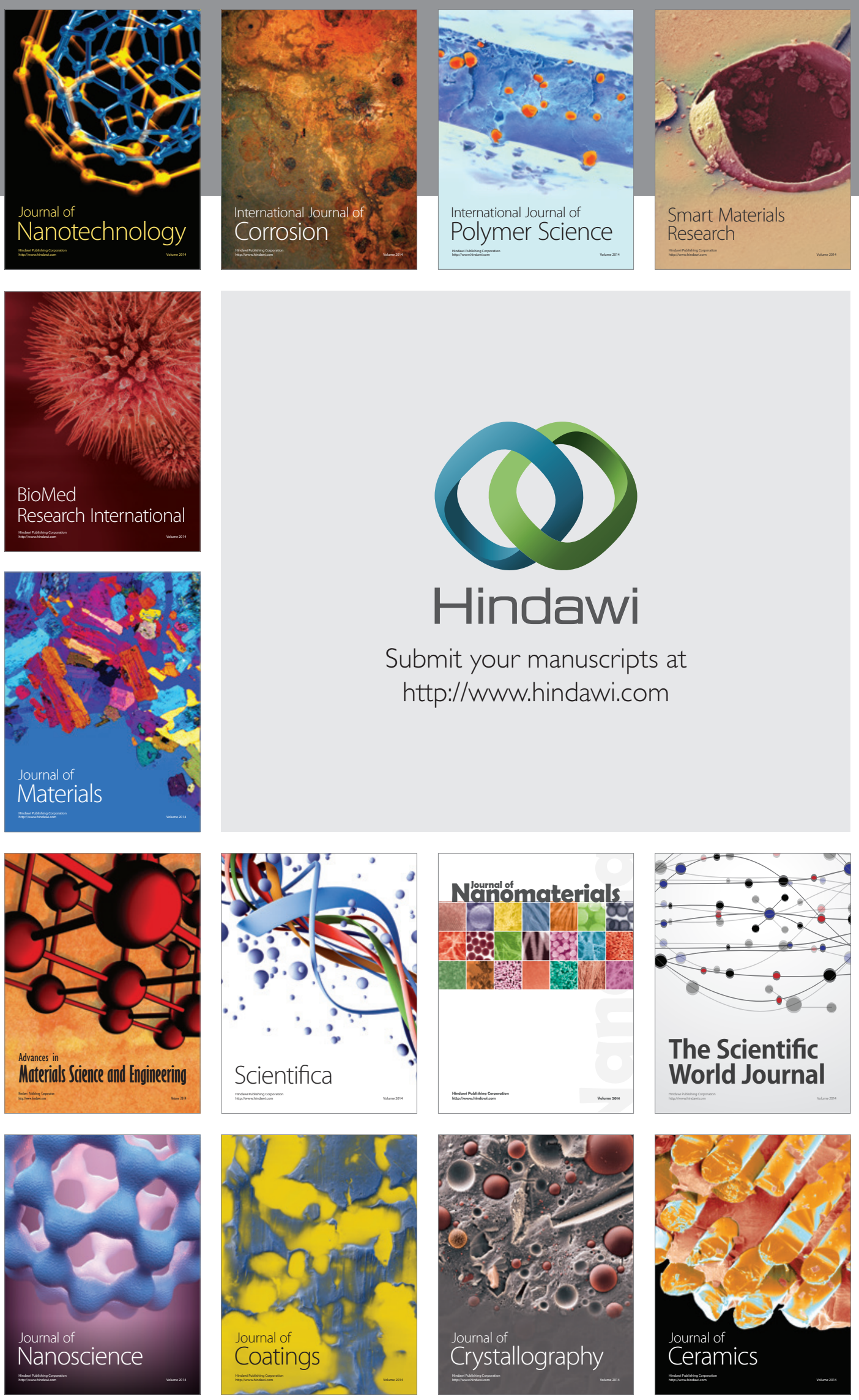

The Scientific World Journal

Submit your manuscripts at

http://www.hindawi.com

\section{World Journal}

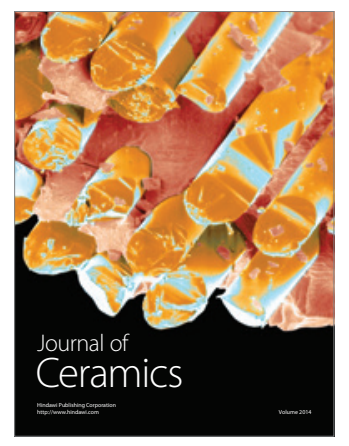

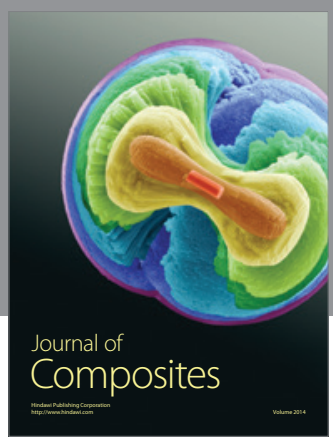
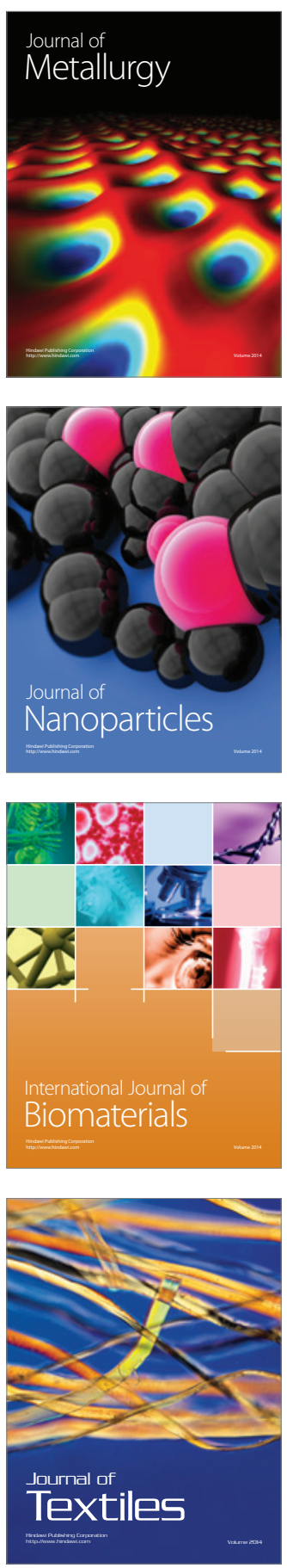